\title{
Rectal Metastatic Adenocarcinoma: Tumor Progression Previous to Liver Metastasectomy
}

Losada B*, Gutierrez D, Juez I, de Torres MV, Pantin MC and Guerra JA

Hospital University of Fuenlabrada, Madrid, Spain

\section{Introduction}

In the evolution of colorectal cancer (CRC), 50\% are metastatic (25\% at baseline and $33 \%$ as relapse), being the liver the most frequent site with a 5 -year survival of $13 \%$. Multidisciplinary management and defining resectability criteria are fundamental strategies for treatment.

\section{Clinical Case}

A 49-year-old man goes to the emergency room for tenesmus and an increasing number of stools. He is admitted and following tests are performed (September 2012).

\section{Analysis}

CEA 21 (Range 4-18), Ca 19.9 7.66 (Range 1-10).

\section{Colonoscopy}

Proliferative lesion at $4 \mathrm{~cm}$ of anal margin. Pathologic result: moderately differentiated adenocarcinoma.

\section{MRI pelvis}

$5 \mathrm{~cm}$ mass from the anal margin with perirectal fat infiltration (T3). $1 \mathrm{~cm}$ adenopathies surrounding the lesion (N1). Liver metastases in segment VI $(24.13 \mathrm{~mm}), \mathrm{V}(6 \mathrm{~mm})$ and VII $(20 \mathrm{~mm})$.

\section{PET-CT}

Rectal tumor with metabolic activity SUV 11.6. Liver affected with two lesions at segment VII of $24 \mathrm{~mm}$ and $13 \mathrm{~mm}$. So with these dates, diagnosis of adenocarcinoma of middle/lower third of rectum stage IV (hepatic cT3N1M1).

\section{We tried to answer the following questions}

Liver or rectal surgery at the beginning? Chemotherapy? The answer for that is that in the case of $>1$ hepatic metastasis $>2 \mathrm{~cm}$ (ESMO Scenarium 0), chemotherapy can increase resectability and response rate and tumor usually do not progress within treatment. In case of progression, many of them are resectable.

After chemo: liver surgery and then rectal surgery or the opposite? We decided hepactectomy because the poor prognosis is conditioned by the metastatic disease. Also non obstructive clinic so rectal surgery is not prioritary. So we started induction chemotherapy in OctoberNovember 2012 (mFOLFOX- $6 \times 3$ cycles), with CEA in descending. CT abdomen: 2 lesions suggestive of metastasis in sg VI of 11 and $15 \mathrm{~mm}$, respectively. As response, we decided hepatectomy 04/01/13 (SgVI-VII) AP: Metatasis Of adenocarcinoma moderadamentally differentiated congruent with origin colorrectal.margins clear. kras native. To achieve a better local control of the disease, we performed RT (25G and including mesorectum, presacro and internal iliacs) followed by rectal amputation (12/02/13): Adenocarcinoma moderately differentiated pT3N1 (1/22) R0. However, tumor progression previous to adjuvant chemotherapy (PET-CT): hepatic tumor progression in segment VI, VII and IV. As they were resectable lesions, we tried to achieve a good response with chemotherapy and then thinking about surgery if no progression. FOLFIRI+Cetuximab $\times 4$ cycles (up to 05/06/2013). CEA 2.2, CA 19.9 12. PET/CT: hepatic metabolic response. Resection segment IVb+Radiofrequency of 2 LOEs segment VI and VII.AP: metastasis with $60 \%$ of persistence in segment IVb. Margins clear. FOLFIRI adjuvant (24/06-06/08/13). Stopping cetuximab in form of rash and diarrhea. PET-CT October 2013 without residual tumor. At November 2015 (more than 2 years without chemo), lung recurrence, retreating with FOLFIRI+Cetuximab, and stable disease nowadays.

\section{Discussion}

4 groups of the ESMO guide: we propose surgery or initial QT according to criteria of liver resectability ( $>4$ lesions, $>5 \mathrm{~cm}$, bilobar involvement, hepatic remnant $>30 \%$ ). Chemotherapy (without antiangiogenics) or surgery can be performed at group 0 ESMO (resectable hepatic metastasis). But on presenting $>3$ metastases, it is decided chemotherapy (EORTC 40983). Also, when initially resectable there is no demonstrated superiority when adding monoclonal Ac. According to get a good local control, literature (Shin, STOCKHOLM III) explains that RT short cycle (5 days $\times 5$ Gy) is effective in local control and down-staging $0.85 \% \mathrm{R} 0$ resections. Also Adams explains that hepatectomy has a 5 -year survival of $8 \%$ against tumor progression vs. 37 and $30 \%$ in response and stabilization. A good strategy is FOLFIRI+Cetuximab in KRAS NATIVE and left colon (not so clear in right colon according to last results in ESMO 2016 and ASCO 2017). But how long to mantain chemotherapy? Cunningham (OPTIMOX) explains that there is no difference in survival from treatment to progression or definite duration. Selection based on prognostic factors and tumor response (early acne in cetuximab OS 26.4 vs. 19.1 months) is necessary.

*Corresponding author: Losada B, Hospital University of Fuenlabrada, Calle Camino del Molino, 2, 28942 Fuenlabrada, Madrid, Spain, Tel: 677098761; E-mail: beatriz.losada@salud.madrid.org

Received June 29, 2017; Accepted September 14, 2017; Published September 20, 2017

Citation: Losada B, Gutierrez D, Juez I, de Torres MV, Pantin MC, et al. (2017) Rectal Metastatic Adenocarcinoma: Tumor Progression Previous to Liver Metastasectomy. J Blood Lymph 7: 179. doi: 10.4172/2165-7831.1000179

Copyright: $\odot 2017$ Losada B, et al. This is an open-access article distributed under the terms of the Creative Commons Attribution License, which permits unrestricted use, distribution, and reproduction in any medium, provided the original author and source are credited. 